\title{
PAUL LEUILLIOT (1897-1987)
}

Paul Leuilliot est mort le 30 mai 1987. Il allait avoir 90 ans. Le Comité de Direction et l'ensemble des collaborateurs des Annales tiennent à saluer sa mémoire et, en demandant à Jean-Jacques Hémardinquer qui lui fut proche de retracer les grandes lignes de sa carrière, à rappeler quel fut son rôle à l'intérieur de la revue et à côté d'elle.

Les Annales

Le dernier membre de l'équipe fondatrice des Annales s'est éteint le 30 mai, à Strasbourg, " berceau » de notre revue. Paul Leuilliot était Normand (et il lui revint de présenter dans les Annales... de Normandie, la mémorable enquête de l'Unesco menée par Lucien Bernot et orientée par Lucien Febvre sur Un village français, Nouville (1952) - en réalité, Nesle-Normandeuse, non loin du collège d'Eu où la vocation de ce fils de greffier s'était dessinée. Mais la guerre de 19141918 lui avait fait adopter l'Alsace.

De la Sorbonne, il arrivait dans la nouvelle université française de Strasbourg, " toute claquante de drapeaux » (L. Febvre), avec une recommandation de Seignobos pour un autre collaborateur de Lavisse, Georges Pariset. Il devait toujours retenir une formule de celui-ci : "C'est une des caractéristiques de la Révolution que la vie locale y est infiniment diverse et que les villes et les pays, même les plus petits, n'ont pas leur histoire identique. " Nommé maître répétiteur au lycée Bartholdi de Colmar, il présenta pour son diplôme d'études supérieures une édition des registres des Jacobins de Colmar. Ce gros travail, publié par la Faculté des Lettres de Strasbourg dès 1923, servit aussitôt, malgré les coupures et la modernisation de l'orthographe, à l'Histoire de la langue française de Ferdinand Brunot. Nommé alors à Strasbourg, le jeune historien y ren-

Annales ESC, novembre-décembre 1987, $n^{\circ}$ 6, pp. 1251-1253. 
contre, comme agrégatif, les maîtres dont il restera le « fidèle » : Marc Bloch et surtout Lucien Febvre.

C'est ainsi qu'il devient secrétaire de la rédaction dès le lancement de leurs Annales, en 1929. Quatre ans après, nommé professeur au lycée Charlemagne, il suit Febvre à Paris, ce qui ne l'empêche pas complètement de poursuivre ses recherches sur l'Alsace - surtout désormais sur la période de la Restauration. En 1939-1940 d'abord évacué avec son lycée à Bordeaux, puis mobilisé en Lorraine, où Bloch lui envoie sa Société féodale, c'est au retour dans Paris occupé qu'il s'affirme une fois de plus « le fidèle Leuilliot » de Febvre : celui-ci, arrachant l'accord de son associé, tient à faire reparaître les Annales sous les deux seuls noms avouables : le sien et celui du secrétaire - qui doit fournir aux autorités allemandes les preuves généalogiques de sa pure aryanité (cf. P. Leuilliot, "Témoignage d'un " fidèle", ", dans $A$ u berceau des Annales, Ch.-O. Carbonell G. Livet éds, p. 71). Son dévouement et sa « bonne expérience » permettent à Febvre, dans les années de reconstruction de l'après-guerre, de lui faire trousser en deux mois un bulletin bibliographique ronéotypé (non continué, semble-t-il) à l'attention des historiens économistes italiens qui avaient perdu le contact. Enfin, en 1951, il l'appelle à l'École Pratique des Hautes Études. Leuilliot y sera le seul ancien élève du fondateur parmi les directeurs d'études de la VIe Section.

C'est tardivement aussi qu'il soutient en 1956 et publie en 1959-1960, sa thèse sur L'Alsace au début $d u X I X^{e}$ siècle, modestement sous-titrée Essai d'histoire politique, économique et religieuse. On n'y retrouve ni la trilogie exacte des Annales, ni aucune concession aux techniques nouvelles... En conclusion, il invite, quoique « tout soit mêlé à tout » (Michelet), à chercher la « clé » de l'histoire dans les structures sociales. Il s'intéresse au recrutement social de la franc-maçonnerie, et on lui doit - comme enseignant et comme membre de la commission d'histoire économique de la Révolution - la publication des répertoires d'A. Le Bihan. D'autre part, il entretient des relations suivies avec deux historiens économistes de Manchester, W. H. Chaloner et W. O. Henderson; il s'associe à l'enquête sur l'indiennage du directeur du musée de l'impression sur étoffe de Mulhouse (et aussi à l'Histoire de Mulhouse), après un temps de prédilection pour l'histoire rurale. Dans les congrès nationaux des sociétés savantes, vice-président de la Section Histoire moderne et contemporaine du Comité des travaux historiques et scientifiques, il organise entre autres les colloques d'histoire de la sécurité sociale. A cette «active curiosité ", sa " défense et illustration de l'histoire locale » et régionale - dont l'écho passe les frontières - apportait un principe d'unité. Dans une série d'articles des Annales ESC sur les problèmes de la recherche (1964-1966), il préconise la création d'un bureau des sociétés savantes au ministère de l'Enseignement supérieur.

Grâce à cette activité multiple, à ses lectures inlassables, le stylo à la main, Paul Leuilliot était devenu une bibliographie vivante. Il avait excellé dans les Annales, à résumer en deux pages un groupe d'ouvrages et articles dont les seuls titres occupaient une demi-page. Son séminaire à l'École des Hautes Études, poursuivi jusqu'en 1978 - à quatre-vingts ans - était en partie consacré à une revue bibliographique et à l'analyse détaillée d'ouvrages parfois encore inédits. On venait le consulter de loin. Venu le triste temps de l'affaiblissement phy- 
sique et du veuvage, il choisit de faire don de sa riche bibliothèque et de ses papiers - des notes prises aux cours de Marc Bloch en 1928 au manuscrit inachevé d'une Alsace sous le Second Empire - à la ville de ses Jacobins : " C'est là que tout commence ", dit un de ses amis d'Alsace, le doyen Livet, témoin d'une cérémonie émouvante, « c'est là que tout finit ». Mais il avait déjà laissé aux Annales, dans les Mélanges en l'honneur de Fernand Braudel, le meilleur historique de leurs origines.

Jean-Jacques HÉMARDINQUER 